\title{
Lab Exercises for a Discrete Structures Course: Exploring Logic and Relational Algebra with Alloy*
}

\author{
Laura E. Brown \\ Michigan Technological University \\ Houghton, MI, USA \\ lebrown@mtu.edu
}

\author{
Adam Feltz \\ Michigan Technological University \\ Houghton, MI, USA \\ adfeltz@mtu.edu
}

\author{
Charles Wallace \\ Michigan Technological University \\ Houghton, MI, USA \\ wallace@mtu.edu
}

\begin{abstract}
Students in computing disciplines need a strong basis in the fundamentals of discrete mathematics, but traditional offline approaches to teaching this material provide limited opportunities for the kind of interactive learning that computing students experience in their programming assignments. We have been using the Alloy language and analyzer to teach concepts in discrete structures (relational algebra, logic, and graphs) in an exploratory, programming-oriented way. Alloy, however, constitutes a new programming paradigm for introductory students, and careful mediation is needed to keep students on track. We use the familiar programming lab format, where students work on small-scope problems co-located with instructors, to provide guidance as students wrestle with the languages of relational algebra and predicate logic through Alloy. We describe selected lab exercises, and report on initial findings based on our experiences with students.
\end{abstract}

\section{CCS CONCEPTS}

- Mathematics of computing $\rightarrow$ Discrete mathematics; Solvers; - Social and professional topics $\rightarrow$ Computing education; • Theory of computation $\rightarrow$ Logic; Constraint and logic programming;

\section{KEYWORDS}

Computer science education, Discrete structures, Logic programming, Alloy

\section{ACM Reference Format:}

Laura E. Brown, Adam Feltz, and Charles Wallace. 2018. Lab Exercises for a Discrete Structures Course: Exploring Logic and Relational Algebra with Alloy. In Proceedings of 23rd Annual ACM Conference on Innovation and Technology in Computer Science Education (ITiCSE'18). ACM, New York, NY, USA, 6 pages. https://doi.org/10.1145/3197091.3197127

\section{INTRODUCTION}

The field of discrete mathematics provides both a formal underpinning and a vital analytical tool set for computing disciplines.

\footnotetext{
${ }^{*}$ This work is supported by the National Science Foundation under grant DUE-1504860

Permission to make digital or hard copies of all or part of this work for personal or classroom use is granted without fee provided that copies are not made or distributed for profit or commercial advantage and that copies bear this notice and the full citation on the first page. Copyrights for components of this work owned by others than ACM must be honored. Abstracting with credit is permitted. To copy otherwise, or republish, to post on servers or to redistribute to lists, requires prior specific permission and/or a fee. Request permissions from permissions@acm.org.

ITiCSE'18, fuly 2-4, 2018, Larnaca, Cyprus

(C) 2018 Association for Computing Machinery.

ACM ISBN 978-1-4503-5707-4/18/07 .\$15.00

https://doi.org/10.1145/3197091.3197127
}

Students in computer science, software engineering, and related programs need a foundation in topics like logic, proof, graph theory, and combinatorics. In many undergraduate computer science programs, these tools are presented in a specialized departmental course, often named "Discrete Structures" as a counterpart to the traditional "Data Structures" course. The core position of Discrete Structures in a computer science education can be seen in the 2013 ACM/IEEE model Computer Science curricula [18]; with 43 total credit hours, it is surpassed only by "Software Development Fundamentals" (with 47 hours).

We argue that bringing an interactive programming spirit to a Discrete Structures course is valuable for two reasons. First, problem solving through the medium of the machine is the essence of computer science. Our majors enjoy engaging with computers, and any course in our curriculum should take advantage of this intrinsic motivation. Second, a programming approach in the course affords active learning. Students are building a conceptual model of the elements of Discrete Structures; through feedback on the soundness of their model, they gain ownership of their own learning.

In our Discrete Structures course, we use the modeling language Alloy and the Alloy Analyzer ${ }^{1}$ to provide hands-on experience with automated feedback. In this paper, we report on our experiences with Alloy in a programming lab context. We cover the basics of the Alloy language and analyzer and describe a lab assignment in predicate logic in some detail ( $(2)$. We then describe the structure of the lab exercises and report on common interactions that happen in lab $(\S 3)$. We cover related work in bringing interaction to Discrete Structures through digital tools $(\S 4)$. We report on the ongoing study of the pedagogical value of these interactive exercises $(\S 5)$ and on qualitative student feedback (§6).

\section{ALLOY-BASED LOGIC EXERCISES}

In the spirit of the name "Alloy", the logic at the heart of this tool combines the quantifiers of predicate logic with the operators of the relational calculus to provide a highly expressive specification language [17]. An Alloy program asserts a set of constraints on mathematical structures over a program-specified signature. The Alloy Analyzer searches within program-specified bounds for models satisfying an Alloy program, through a SAT-based constraint solver. If a satisfying model is found, the analyzer displays it graphically; otherwise, it identifies a minimal unsatisfiable core of the program. Alloy has been used in a wide array of application areas, including security, computer architecture, program verification, and model driven development. It has also been used as a teaching tool, but

$\overline{{ }^{1} \text { http://alloy.mit.edu }}$ 
reports of its use focus solely on more advanced topics in formal methods or software modeling $[1,4,30]$.

Alloy's expressive flexibility allows us to use it for a variety of topics. Constraints may be expressed in Alloy as first-order formulas, as relational expressions, or a combination of both. This flexibility provides an opportunity to illustrate a conceptual continuum in the course material. Starting with sets and relations, we state Alloy constraints as relational expressions. Later, when we cover first-order logic, we introduce this other style of expression in Alloy and show how it compares to the relational style. Finally, we address problems in graph theory using both styles, taking advantage of the Alloy Analyzer's visualization functionality.

The feedback provided by the Alloy Analyzer has the potential to eliminate common misconceptions among students. Compared to a traditional approach where students simply submit written answers to homework problems, students working on Alloy problems get immediate critique of the wellformedness and satisfiability of their responses. A common student response during lab exercises is that they "don't understand Alloy". Experience has shown, however, that the root of the problem, is rarely the Alloy language, since its structure is so close to that of standard quantified formulas and relational expressions. Working through such student problems usually reveals that they have a misunderstanding of the structure of standard mathematical notation. With a traditional pencil-andpaper exercise, students can remain in a false state of confidence, and deficiencies in understanding are not exposed until after the exercise is submitted for grading.

Students can engage with Alloy at various levels of sophistication. For our early Discrete Structures course, we do not expect students to build sophisticated Alloy code from scratch. As described in an earlier paper, we design exercises carefully to take students from observers of program behavior to tweakers of search parameters and builders of more substantial constraints [31].

In the following examples, we concentrate on exercises in predicate logic, exploring the possibilities of love relationships between sentient beings, through a binary "love" relation between "kittehs".

\subsection{Observing}

One useful observation exercise is to explore the hazards of syntactically correct and even satisfiable predicate logic formulas that, despite appearances, do not represent the intended constraint. The example in Fig. 1, taken from Jackson [17], appears to be an accurate translation of the song title "Everybody Loves My Baby, but My Baby Don't Love Nobody but Me". ${ }^{2}$ The first line declares a binary love relation over the signature Kitteh. The expression $\mathrm{k} 2$ in $\mathrm{k} 1$. love signifies that there is a pair $\mathrm{k} 1->\mathrm{k} 2$ in the love relation; the predicate inLove with parameters $k 1$ and $k 2$ provides a slightly more succinct way to express the same proposition.

The predicate myBaby with parameters me and baby specifies a constraint, expressed in predicate logic, on satisfying models. In the formula, some and all represent the quantifiers $\exists$ and $\forall$, respectively, and $\mathrm{k}$ is a bound variable. The formula asserts that baby is loved by all Kittehs (line 2), and asserts that me is the only Kitteh loved by baby (line 3). The final line limits the search for satisfying models to those with no more than $4 \mathrm{Kittehs.}$

\footnotetext{
${ }^{2}$ Assuming everybody, including my baby and me, are Kittehs.
}
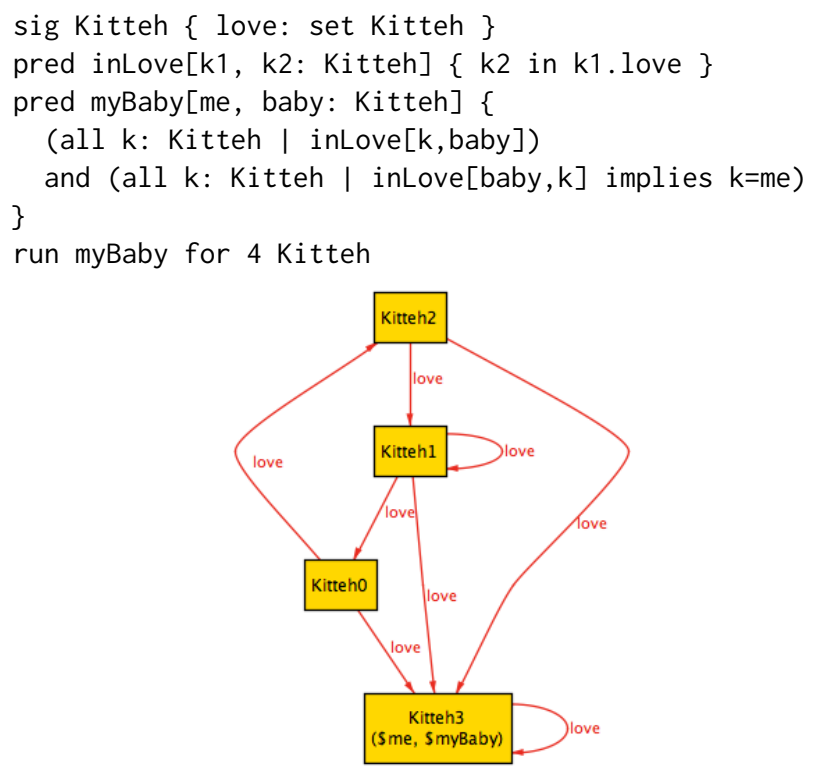

Figure 1: The myBaby example, and a satisfying model.

Students are given the code and asked to find the commonality among all satisfying models: does the formula capture the meaning of the song title? The Analyzer generates a series of visualizations of satisfying models, like the one in Fig. 1. To their surprise, the only satisfying models found are ones in which baby and me are identical! (In Fig. 1, Kitteh3 is both me and baby.) The desired meaning can be achieved by adding an assertion that baby and me are not the same, plus removing baby from the scope of the first all quantifier.

\subsection{Tweaking}

Starting with a complete Alloy program, both the constraints and the scope of the search for satisfying models are easily modified. This allows for interesting explorations based on established code. Such scaffolding gives students a safe entry into Alloy syntax, where they can focus on the portion relevant to logic. A simple example is shown in Fig. 2: What difference does it make if the order of universal and existential quantifiers are swapped in a formula? In formula (1), the wide scope of the universal quantifier makes a claim about all kittehs: each loves at least one kitteh (including possibly itself). Students can run the Alloy code and observe a series of models like the one illustrated, noting the commonality between them. Switching the order of quantifiers, as in (2), results in a claim about a particular kitteh: it loves all kittehs. The satisfying models generated, for instance the one illustrated, have a clearly visible commonality. The tweaking exercise emphasizes the fact that small syntactic changes to a formula can have big effects.

\subsection{Building}

After sufficient exposure, and along with similar supplementary examples, students are ready to build their own formulas. Following 
(1) pred allLoveSome \{

$$
\text { all k: Kitteh I some k': Kitteh । }
$$$$
\text { inLove[k, } \left.k^{\prime}\right]
$$

\}

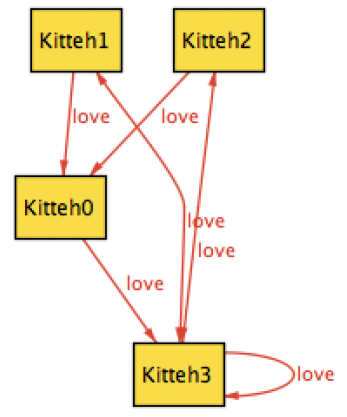

(2) pred someLoveAll \{

some k: Kitteh | all k': Kitteh |

inLove[k, $\left.k^{\prime}\right]$

\}

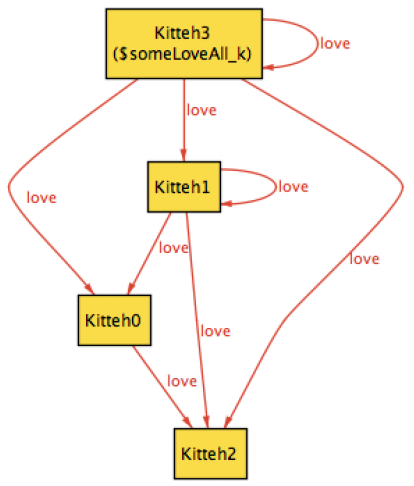

Figure 2: Tweaking a formula.

on the previous example, students are asked to translate various "kitteh love" scenarios into predicate logic, sample solutions in Fig. 3: Narcissists Every kitteh loves himself but loves no other kitteh. Love Story Every kitteh has another kitteh who he loves and who loves him back.

Rivals There are two different kittehs who love the same kitteh, but who don't love each other.

Sociopath There is a kitteh who all other kittehs love, but who loves no kitteh except himself.

\section{LAB ASSIGNMENTS}

Our Discrete Structures course is intended for Computer Science, Software Engineering, and Computer Engineering majors. Enrollment each term runs from 120 to 150 . Computer Science and Software Engineering majors typically take it in the second or third term; Computer Engineering majors tend to take it a year later. The students in this course are facing a great deal of new material, including the new languages of relational algebra and predicate logic. A concern is that a high-powered tool like Alloy in the hands of students at an early stage of development can be dangerous. The

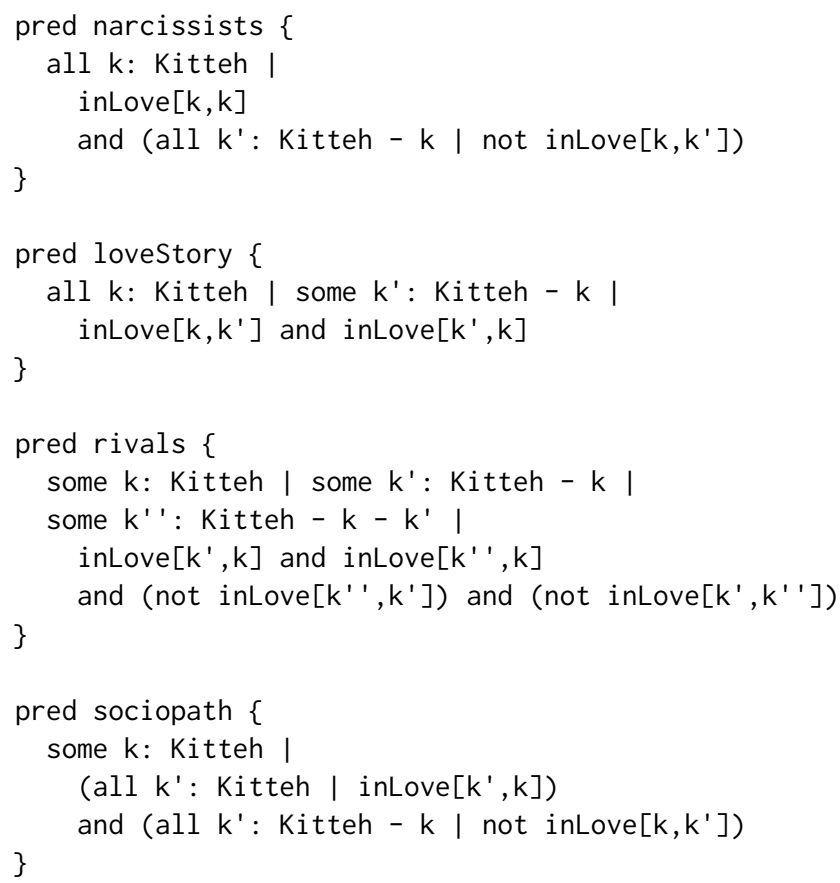

Figure 3: Kitteh love predicates in Alloy.

declarative nature of Alloy is a conceptual departure from their still nascent conception of what programming is. Alloy does not look or behave like the "CS1" language (in our case, Java) they have only recently been exposed to, and they can easily fall into despair at learning an entirely new language and a new style of programming.

Given that the standard mathematical notation for sets, relations, propositions, and quantifiers is new material for them, the mapping between this notation and Alloy syntax must be as transparent and direct as possible-and we believe that it is. Furthermore, it is important to provide scaffolding for early concept formation. The use of a quick reference guide, that distills the traditional mathematical notation into a single page with translations into Alloy, has been extremely useful, and in fact students use the "cheat sheet" even when working strictly within traditional syntax, since it neatly summarizes the tool set at their disposal.

Prior experience indicates that students can falter and become alienated when working on Alloy programs in isolation [31]. The debugging strategies they have just started to develop for imperative programs do not necessarily work with Alloy, and a common (though highly deprecated) strategy among novice programmers searching for similar code online - works for a language like Java with a huge code base, but not so well with Alloy. We have mitigated this risk by introducing a lab component to the Discrete Structures course, similar to labs in traditional introductory programming courses. In this format, the instructor can intervene at crucial moments and provide guidance. Through appropriate scaffolding and instructor mediation, we are able to maintain student focus on what is relevant - logic and relational algebra - and less on the details of the Alloy language and programming environment. 


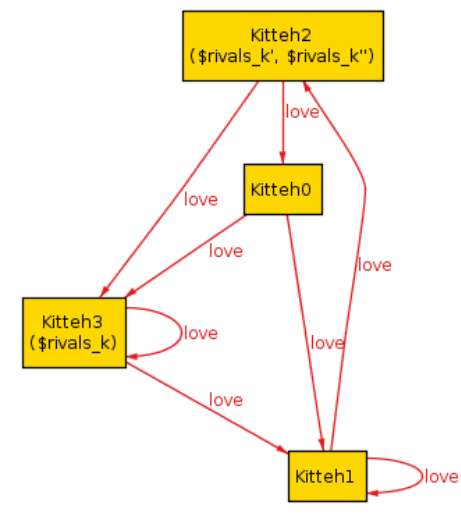

Figure 4: Catching a bug in the rivals problem.

Alloy lab exercises are conducted in a fashion similar to the labs of our introductory Java programming course [33]. In sections of 30 to 40 each, students co-located in a programming lab space work in pairs on problems using the Alloy software and submitting answers through the Canvas ${ }^{3}$ online quiz function. The instructor acts as lab assistant, coaching students through the assignments. Each lab runs for 75 minutes; the majority of students are typically able to complete the assignments on time, but those who are not were allowed to complete them within 48 hours.

A common mistake in the rivals problem is to forget to restrict the scope of the third existential quantifier (binding $k$ ' ') to exclude $k '$. Students making this mistake execute their code and encounter models like that shown in Fig. 4. Here the beloved Kitteh3 has both Kitteh 0 and Kitteh2 as admirers, but there is a love relationship between the "rivals". What is happening here? The visualization has the answer: both k' and k' ' have been instantiated as Kitteh2, contrary to the stipulation that the rivals be distinct.

Within the Kitteh Love problem set, after students define the relevant predicates, we can ask exploratory questions that can be readily answered through appropriate tweaking. For instance: is it possible for all Kittehs to be sociopaths? The question is easily answered by modifying the rivals predicate, replacing the some quantifier with all, then executing the result. The Analyzer reports that the predicate is satisfiable, but that there are only two satisfying models: one with zero Kittehs, the other with one Kitteh.

In addition to the KittehLove exercise described earlier, three other exercises were conducted:

\subsection{Food web (relational algebra)}

A small ecosystem is modeled in terms of a binary eats relation over a signature Organism. Students translate terms like "top predator" (organisms eaten by no organism) using the standard relational operators built into Alloy. They also use the transitive closure operator, which is also predefined in Alloy, to capture chains of food web relations. In this early lab, students simply evaluate relational expressions in the context of a fixed food web model.

\footnotetext{
${ }^{3} \mathrm{http}: / /$ www.instructure.com
}

\subsection{Synchronization models (partial orders)}

Program executions are modeled in terms of a binary "happensbefore" temporal relation over a signature of operations. Students start with a serial model of computation where the temporal order is total, then define less strict models such as sequential consistency, where the relation is partial but not total. Here students move into building new constraints in the Alloy language.

\subsection{Graph structures}

Students define graph structures such as clique and dominating set in Alloy, then verify their solutions by running the Analyzer. Once the definitions are in place, they can use the Analyzer to answer questions about these structures. For instance: can a clique of size greater than 1 also be an independent set? (Answer: no.) Can it also be a dominating set? (Answer: yes; Alloy provides an example with 4 vertices.) Here the Alloy Analyzer reveals valid but unexpected results that challenge the students' assumptions.

\section{RELATED WORK}

Our use of Alloy in an introductory discrete mathematics course appears to be novel. Most Discrete Structures textbooks include programming problems related to each topic, however, the exercises do not come with a common framework or scaffolding [20,27]. Other books relate Discrete Structures to topics from software engineering [11-13]. A few books and papers have advocated for teaching discrete mathematics and functional programming together [32], with Haskell used for programming projects $[7,13]$. The Beseme Project bridges these ideas by structuring the teaching of Discrete Structures via a software-centric approach [13, 24].

Many learning tools have been developed for use in Discrete Structures that employ interactive components to give students visual representation of the problems, immediate feedback (correctness, explanatory, etc.) or gamification to challenge and engage student learners $[5,6,10]$. A majority of learning tools focus on a specific topic of a Discrete Structures course. For example, finite automata has tools such as, Pushdown Automata Simulator [14], JFLAP [26], ASSIST [15]. Logic and proofs has the greatest number of tools created [3], e.g., Boole and Fitch [2], Logic-ITA [35], Proof Web [16], ORGANON [8], AProS - automated proof search's Proof Lab and Proof Tutor [28, 29], FOLST and LogicChess [22], and Connect the Dots [23].

A longstanding learning tool Tarski's World [2] has a similar intended audience and similar aims to ours, but operates within a more restricted language that avoids some pitfalls-but also some potential benefits-of Alloy's greater expressivity. Students using Tarski's World operate in the opposite direction from Alloy, by first constructing a model (using predefined sets and relations) and then writing predicate logic formulas describing the model, using the software's automated verification to test which formulas are satisfied by the model. In contrast, students using Alloy construct formulas and then use the Analyzer to find satisfying models. An advantage of the Alloy approach is that students are confronted with unexpected "corner cases": models that satisfy the given constraints in unanticipated ways. Furthermore, the richer language at the heart of Alloy allows us to explore a variety of application domains and sophisticated relational operators like transitive closure. 


\begin{tabular}{lccccccc}
\hline & 1 & 2 & 3 & 4 & 5 & 6 & 7 \\
\hline 1. Score & 1 & & & & & & \\
2. Confidence & $.44^{* *}$ & 1 & & & & & \\
3. Success & .16 & $.42^{* *}$ & 1 & & & & \\
4. Usefulness & $.25^{*}$ & $.52^{* *}$ & $.48^{* *}$ & 1 & & & \\
5. Motivation & $.33^{*}$ & $.49^{* *}$ & .17 & $.58^{*}$ & 1 & & \\
6. Self-Efficacy & .21 & $.64^{* *}$ & $.27^{*}$ & $.38^{*}$ & $.43^{* *}$ & 1 & \\
7. Grit & -.11 & 0 & .23 & .16 & .16 & .05 & 1 \\
\hline${ }^{*} p<.05,^{* *} p<.01$, two-tailed & & & & &
\end{tabular}

Table 1: Correlations of predictor variables and outcome variables, Study 1.

\section{PILOT STUDY ANALYSIS AND RESULTS}

There is good theoretical reason to think that the Alloy tool will improve student performance with discrete structures [21, 25]. We plan on verifying our theoretical predictions with empirical evidence from controlled studies.

We already have some suggestive empirical evidence from a pilot study, with one group of students in a Discrete Structures class that did not use Alloy $(N=62)$, and a separate group of students in a Discrete Structures class that used Alloy $(N=122)$. The main comparison was between student performance on similar test items (scores were converted to a 100 point scale). The course with the Alloy tool $(M=73.39, S D=16.12)$ had significantly better scores than the class without the Alloy tool $(M=63.11, S D=$ $16.88), F(1,183)=16.12, p<.001, d=.63$. These results should be treated with caution, however, because the test items were similar but not identical. The test item for the Alloy class could have been easier or more easily graded than the test items in the non-Alloy class. For more direct comparisons, future research will pair identical test item and have more than one rater evaluate the items to determine inter-rater reliability.

If the Alloy tool does improve performance, it is also important to identify how it improves performance. Theory suggests that there are likely some predictors of student success including confidence, motivation, how useful the material is thought to be [34], and selfefficacy $[9,19]$. In the pilot study, we had participants in the Alloy class complete a survey that measured those factors at the beginning of the semester. As we hypothesized, confidence, usefulness, motivation, and self-efficacy would predict performance in the class (correlation matrix reported in Table 1).

The data from the pilot study suggested that Alloy improves performance and that we can model some of the ways that improvement happens (e.g., by increasing self-efficacy). Given these data, we plan on performing a controlled experiment with a longitudinal design. We have identified one class that uses Alloy and one class that does not. Our study will determine the effectiveness of the Alloy tool by having both groups complete the same skill-based materials in discrete structures. Our primary goal is to evaluate students on these tasks to see if Alloy improves performance. Our secondary goal is to determine how the Alloy tool improves performance. This will be accomplished by measuring students' confidence, beliefs about discrete structures' usefulness, motivation in the class, and computer-science self efficacy in a pre/post-test design. Our prediction is that the Alloy tool will increase performance by increasing

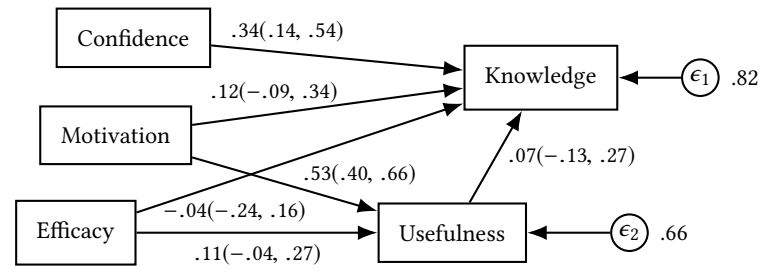

Figure 5: Structural model predicting performance.

students' motivation, self-efficacy, and confidence (see Fig. 5 for our hypothesized structural model). The ways in which Alloy likely improves performance are desirable ends in themselves since students' motivation, self-efficacy, and confidence are qualities that education in general attempts to promote.

\section{QUALITATIVE RESULTS}

Student provided feedback on their Alloy lab experiences through freeform comments in their course evaluations. A total of $78 \mathrm{stu}-$ dents out of 136 completed the online evaluation. There were 89 responses to these two prompts; of these, 34 specifically mentioned the lab exercises. It should be mentioned that there was one additional lab that used the JFLAP tool [26] to construct and execute finite-state automata, so general comments about labs should be interpreted as covering both Alloy and JFLAP labs.

The prompts for open comments are the following: "As I, the instructor, prepare to teach this class again, what aspects of this course (teaching methods, assignments, areas of emphasis, etc.) should I preserve that effectively furthered your learning?" and "What aspects of this course should I change to improve student learning? Specifically, what would you suggest?"

Of the 34 relevant responses, 26 were positive about the lab exercises. The following representative comments indicate that the approach is valued by a substantial number of students:

The labs were a great experience. They forced you to sit down and try to solve the problems with a partner while you were there to help. I would say that the labs were easily where I learned the most.

The labs were helpful to be able to visualize things. I liked how in the labs you didn't just give us the answers if we didn't understand it. Instead you gave us hints to help us see the answers ourselves.

The labs were helpful for understanding what we were learning about in class and applying topics to situations.

Having the labs (even though they were frustrating for me), I think helped me understand many of the topics of discrete structures better.

The positive comments suggest that the labs are challenging students in ways they acknowledge as painful but rewarding; that the visualization of results strengthens understanding; and that the applied nature of the exercises increases their perceived relevance.

Five comments were explicitly negative about the value of the lab exercises in general or negative about the Alloy labs in particular. Of this set, only one comment went into detail:

I'm very conflicted about the lab. I'm not sure if it really enforced my learning of the material. Although, the lab with the automata was incredibly useful and a great learning booster, as it's the exact

${ }^{4}$ The JFLAP lab. 
way you actually implement state-logic type of material. The rest of the labs were more of "learn how to do the same problems in this strange language" rather than "this will bolster your knowledge of the material".

The student raises an important question: to what degree are we conveying the fact that Alloy syntax is a close match to traditional mathematical notation? Although the mapping from Alloy to standard notation is remarkably direct, perhaps there is a transfer issue at play. Alternatively, perhaps it is the language of mathematics that is the "strange language", not considered relevant to the student's personal learning goals.

Three comments addressed the opportunity cost of using contact hours for lab instead of lecture. The following representative comment addresses an important point:

I felt it wasn't very fair to ask us to come to lab at a given time, when it was not budgeted in our schedules. If the lab is something that's going to stick around it should be an actual course with a TA because there were a lot of questions and only one of you.

Our Alloy labs were necessarily ad hoc in nature, substituting lab time for lecture time, and without graduate teaching assistants. A truly lab-based course should indeed be structured as such, with lab hours built into the official schedule and with appropriate teaching assistant support.

\section{CONCLUSION}

Our initial results indicate that Alloy has promise as a practical tool for students encountering logic and relational algebra for the first time. We are conducting further study, following the design described here ( $\$ 5)$, to confirm the degree of the effect on student learning. We are sanguine about the prospects of a lab-oriented Discrete Structures course, in which Alloy is just one of several interactive learning tools. If a lab component does indeed have measurable benefits, we will consider introducing another credit hour to the course and to schedule lab hours.

It must be acknowledged, however, that this kind of intervention has a high time and labor cost. For many educational settings, it may be unrealistic to assign additional credit hours, not to mention qualified lab assistants, to a Discrete Structures lab. Consequently, we plan to test more modest interventions involving Alloy: for instance, observing and tweaking activities done as part of a lecture. Whatever the right level of commitment turns out to be for a particular course, we feel that the interactive feedback provided by Alloy and other tools can help to make the Discrete Structures course a more effective, authentic, and enjoyable component of a computing curriculum.

\section{REFERENCES}

[1] T. Ball and B. Zorn. 2015. Teach Foundational Language Principles. Commun. ACM 58, 5 (2015), 30-31.

[2] D. Barker-Plummer, J. Barwise, and J. Etchemendy. 2007. Language, Proof and Logic. CSLI Publications.

[3] Patrick Blackburn, Hans van Ditmarsch, María Manzano, and Fernando SolerToscano (Eds.). 2011. Tools for Teaching Logic: Third International Congress TICTTL 2011. Springer Berlin Heidelberg, Berlin, Heidelberg.

[4] R.C. Boyatt and J.E. Sinclair. 2008. Experiences of Teaching a Lightweight Formal Method. In Proceedings of Formal Methods in Computer Science Education.

[5] Fabienne M. Van der Kleij, Remco C. W. Feskens, and Theo J. H. M. Eggen. 2015 Effects of Feedback in a Computer-Based Learning Environment on Students' Learning Outcomes: A Meta-Analysis. Review of Educational Research 85, 4 (2015), 475-511.
[6] Darina Dicheva, Christo Dichev, Gennady Agre, and Galia Angelova. 2015. Gamification in Education: A Systematic Mapping Study. Fournal of Educational Technology \& Society 18, 3 (2015), 75-88.

[7] Kees Doets and Jan van Eijck. 2004. The Haskell Road to Logic, Maths and Programming. College Publications.

[8] Ludmila Dostalova and Jaroslav Lang. 2011. ORGANON: Learning Management System for Basic Logic Courses. In Tools for Teaching Logic, Patrick Blackburn, Hans van Ditmarsch, María Manzano, and Fernando Soler-Toscano (Eds.). Springer Berlin Heidelberg, Berlin, Heidelberg, 46-53.

[9] A. Duckworth, C. Peterson, M. Matthews, and D. Kelly. 2007. Grit: Perserverence and Passion for Long-Term Goals. fournal of Personality and Social Psychology 9 (2007), 1087-1101.

[10] Wolfgang Effelsberg and Stefan Göbel. 2014. Serious Games 2014: International Workshop on Serious Games. In Proceedings of the 22Nd ACM International Conference on Multimedia (MM '14). ACM, New York, NY, USA, 1265-1266.

[11] W. Grassman and J-P. Tremblay. 1996. Logic and Discrete Mathematis: A Computer Science Perspective. Prentice Hall.

[12] D. Gries and F. Schneider. 1993. A Logical Approach to Discrete Math. SpringerVerlag.

[13] Cordilia Hall and John O'Donnell. 2000. Discrete Mathematics Using a Computer. Springer.

[14] Mohamed Hamada. 2009. Pushdown Automata Simulator. In Learning by Playing. Game-based Education System Design and Development, Maiga Chang, Rita Kuo, Kinshuk, Gwo-Dong Chen, and Michitaka Hirose (Eds.). Springer Berlin Heidelberg, Berlin, Heidelberg, 328-338.

[15] Eileen Head. 1998. ASSIST: A Simple Simulator for State Transitions. Ph.D. Dissertation. http://www.cs.binghamton.edu/ software

[16] Maxim Hendriks, Cezary Kaliszyk, Femke Van Raamsdonk, and Freek Wiedijk. 2010. Teaching logic using a state-of-the-art proof assistant. Acta Didactica Napocensia 3, 2 (2010), 35-48.

[17] D. Jackson. 2012. Software Abstractions: Logic, Language and Analysis. MIT Press.

[18] Joint Task Force on Computing Curricula. 2013. Computer Science Curricula 2013: Curriculum Guidelines for Undergraduate Degree Programs in Computer Science. ACM and IEEE Computer Society.

[19] O. Korkmaz and H. Altun. 2014. Adapting computer programming self-efficacy scale and engineering students' self-efficacy perceptions. Participatory Educational Research 1 (2014), 20-31.

[20] Eric Lehman, F. Thomas Leighton, and Albert R. Meyer. 2017. Mathematics for Computer Science. Samurai Media Limited.

[21] Q. Li and X. Ma. 2010. A meta-analysis of the effect of computer technology on school studentsâĂŹ mathematics learning. Educational Psychology Review 22 (2010), 215-243.

[22] Maria Virginia Mauco, Enzo Ferrante, and Laura Felice. 2014. Educational Software for First Order Logic Semantics in Introductory Logic Courses. Information Systems Education fournal 12, 6 (2014), 15-23.

[23] Mark McCartin-Lim, Beverly Park Woolf, and Andrew McGregor. 2018. Connect the Dots to Prove It: A Novel Way to Learn Proof Construction. In Proceedings of the ACM Technical Symposium on Computer Science Education. 533-538.

[24] Beseme Project. 2003. http://www.cs.ou.edu/ beseme/index.html

[25] C. Rakes, J. Valentine, M. McGatha, and R. Ronau. 2010. Methods of instructional improvement in algebra: A systematic review and meta-analysis. Review of Educational Research 80 (2010), 372-400.

[26] Susan Rodger and Thomas Finley. 2006. FFLAP - An Interactive Formal Languages and Automata Package. Jones and Bartlett.

[27] K.H. Rosen. 2012. Discrete Mathematics and Its Applications (7 ed.). McGraw-Hill.

[28] Christian D. Schunn and Mellisa M. Patchan. 2009. An Evaluation of Accelerated Learning in the CMU Open Learning Initiative Course "Logic \& Proofs".

[29] Wilfried Sieg. 2007. The AProS Project: Strategic Thinking \& Computational Logic. Logic Journal of the IGPL 15, 4 (2007), 359-368.

[30] S. Tarkan and V. Sazawal. [n. d.]. Chief Chefs of $Z$ to Alloy: Using a Kitchen Example to Teach Alloy with Z. In Teaching Formal Methods. Number 5846. Springer Lecture Notes in Computer Science, 72-91.

[31] Leo C. Ureel and Charles Wallace. 2016. Discrete Mathematics for Computing Students: A Programming Oriented Approach with Alloy. In Frontiers in Education.

[32] Thomas VanDrunen. 2011. The Case for Teaching Functional Programming in Discrete Math. In Proceedings of the ACM International Conference Companion on Object Oriented Programming Systems Languages and Applications Companion (OOPSLA '11). ACM, New York, NY, USA, 81-86.

[33] Charles Wallace. 2018. Learning Discrete Structures Interactively With Alloy. In Proceedings of the ACM Technical Symposium on Computer Science Education. 1051.

[34] E. Wiebe, L. Williams, K. Yang, and C. Miller. 2003. Computer science attitude survey. http://www4.ncsu.edu/ wiebe/www/articles/prl-tr-2003-1.pdf

[35] Kalina Yacef. 2005. The Logic-ITA in the Classroom: A Medium Scale Experiment. Int. F. Artif. Intell. Ed. 15, 1 (Jan. 2005), 41-62. 